\title{
Programas de avaliação em academias de ginástíca: o que se faz?
}

\author{
Assessment programs in gyms: what \\ usually do?
}

\author{
Ninrodes Muniz de Oliveira \\ Diego Augusto Santos Silva² \\ Jose Jean de Oliveira Toscano'
}

\section{RESUMO}

Objetivou-se analisar o processo estrutural dos programas de avaliação em Academias de Ginástica no município de Maceió-AL. Esse estudo foi do tipo transversal, sendo a amostra constituída por 58 academias. Aplicou-se um questionário com os responsáveis pela avaliação física, contendo as variáveis da rotina de avaliação nas respectivas academias: anamnese, medidas fisiológicas (pressão arterial sistêmica), avaliação postural, aptidão física relacionada a saúde e emissão de laudos. $\mathrm{Na}$ análise dos dados utilizou-se o teste de comparação entre duas proporções, Qui-quadrado de heterogeneidade ou o Teste Exato de Fisher. Foi estabelecido nível de significância de $5 \%$. A maior parte das academias exigia a obrigatoriedade da avaliação física na matrícula $(\mathrm{p}=0,005)$ o que não se observou quanto à reavaliação $\mathrm{p}(=0,079)$; os indicadores presentes na anamnese quanto as morbidades auto referidas, ingestão de medicamentos, tabagismo e atividade física estiveram presentes na rotina de avaliação. Além disso, a mensuração da pressão arterial sistêmica, avaliação postural e composição corporal apresentou presença significativa na rotina do programa de avaliação. Nas academias cuja avaliação era obrigatória, a reavaliação também foi; o objetivo da clientela estava na saúde; a avaliação do componente da aptidão física flexibilidade foi negligenciado. De modo geral, os indicadores presentes no processo de avaliação das academias podem ser mais bem estruturados para que possam apresentar um melhor perfil da população usuária desse serviço.

\section{PALAVRAS-CHAVE}

Academias de ginástica; Avaliação física; Indicadores de saúde; Aptidão física.

\begin{abstract}
The objective of this study was to analyze the structural process of physical assessment of Fitness Centers from Maceió-AL. In this cross-sectional study, 58 Fitness Centers were evaluated. A self-reported questionnaire with information about physical assessment was answered by Physical Education professionals. Comparison Test between two proportions, Chi-square Test and Fisher's exact Test was used to identify associated variables with physical assessment of Fitness Centers. Most of the gyms demanded the mandatory physical assessment on enrollment $(p=0.005)$ which was not observed for the reassessment ( $p=0.079)$; the indicators present in anamnesis as the self-morbidities, medication intake, smoking and physical activity were present in the routine evaluation. Furthermore, measurement of systemic blood pressure, postural and body composition assessment had a significant presence in the routine evaluation program. In the gyms whose assessment was required, the revaluation was also; the goal of the clientele was in health; the assessment of the flexibility was neglected. In general, the indicators present in the process of physical assessment of Fitness Centers should be reorganized so that they can be used effectively.
\end{abstract}

\section{KEYWORDS}

Gyms; Physical assessment; Health indicators; Physical fitness.
Rev Bras Ativ Fis Saúde p. 568-578 DOI

http://dx.doi.org/10.12820/rbafs.v.19n5p568

1 Universidade Federal de Alagoas

2 Universidade Federal de Santa Catarina 


\section{INTRODUÇÃOO}

As evidências na literatura apresentando os numerosos benefícios de um estilo de vida ativo têm como principal objetivo aumentar a participação individual em uma atividade física regular de intensidade moderada a vigorosa, e vem sendo sistematicamente elaboradas e atualizadas por instituições internacionais como o Centers for Disease Control and Prevention (CDC), o American College of Sports Medicine (ACSM) e o American Heart Association (AHA) ${ }^{1}$. Já os esforços para avaliar indivíduos que se matriculam em instalações de saúde/fitness são limitados e inconsistentes².

Nas diretrizes publicadas pelo $\mathrm{ACSM}^{3}$ há duas finalidades que devem ser consideradas quando na participação de indivíduos na prática de atividades físicas: a primeira seria avaliar o risco do indivíduo para eventos adversos relacionados ao exercício, e a outra, elaborar recomendações aprimoradas acerca do início, da continuação ou da progressão de um programa de atividade física.

Para se atingir as referidas finalidades a proposta é que um programa de avaliação possua duas categorias: processo e impacto, onde a primeira investiga se a intervenção ou serviço está sendo ofertado adequadamente, se sua qualidade é apropriada e se os indivíduos estão efetivamente utilizando o serviço; quanto às avaliações de impacto, seriam responsáveis por analisar a evolução de determinados indicadores na população atendida ${ }^{4-5}$.

As academias de ginástica representam um importante segmento de prestação de serviço tanto para oportunizar a população adulta a aumentar o nível de atividade física, quanto para intervenção dos profissionais de Educação Física. De acordo com o Relatório IHRSA Global Report 2012, o Brasil é o segundo país no mundo em número de academias, possui 23.398 academias registradas junto ao Sistema CREF/CONFEF, ficando atrás somente dos Estados Unidos ${ }^{6}$. Apesar da quantidade de indivíduos que participam nas modalidades oferecidas em academias ser baixa, insuficiente para determinar mudanças no estilo de vida populacional, esses centros apresentam potencial para influenciar o estilo de vida da população de modo a reduzir o grau de sedentarismo ${ }^{7}$. A busca da melhoria da aparência física e as preocupações que aparecem cada vez mais associadas à própria noção de saúde fazem parte dos fatores responsáveis pela expansão nesse setor ${ }^{8}$.

Há um destacado crescimento desse campo de atuação profissional, no entanto, existe lacuna de conhecimento quanto ao conteúdo dos programas de avaliação física montada nas academias. Levantamento realizado nas principais bases de dados (Pubmed, Scopus, Scielo) com os termos "Atividade Física", "Avaliação", "Avaliação em Saúde", "Avaliação de Programas", "Avaliação de Serviços de Saúde", "Educação Física e Treinamento", "Musculação", "Treinamento de Resistência" e "Academias de Ginástica", no período de 2000 a Março de 2013 não foi encontrado nenhuma pesquisa que analisasse a estrutura organizacional dos programas de avaliação utilizados em academias de ginástica.

Os profissionais de Educação Física, pelo alcance e pela natureza de seu trabalho, que requer avaliação de grande número de capacidades, qualidades e conhecimentos, necessitam avaliar diferentes indicadores que apresentam relação com a realização de esforços físicos, independente dos seus objetivos, o que requer preocupação em garantir qualidade às informações a serem analisadas, necessitando de planejamento ao que será ofertado à população como sendo avaliação. 
Dessa forma, o objetivo desse estudo foi analisar a estrutura organizacional dos programas de avaliação implantados em academias de ginástica, bem como verificar as diferenças nos programas de avaliação existentes entre academias que têm à avaliação física como obrigatoriedade e aquelas que não exigem a realização da avaliação física no município de Maceió-AL.

\section{MÉTODOS}

O presente estudo se caracterizou como sendo transversal. A população alvo foi composta por academias de ginástica de Maceió-AL, Nordeste do Brasil, representadas pelos profissionais que realizavam avaliação física. $\mathrm{Na}$ delimitação da amostra, foi solicitado à Vigilância Sanitária lista com o quantitativo de academias cadastradas nas sete regiões administrativas do referido município. Ao todo estavam registrados 113 estabelecimentos. Em seguida agruparam-se as academias de acordo com a respectiva região em que se localizavam. (Figura 1).

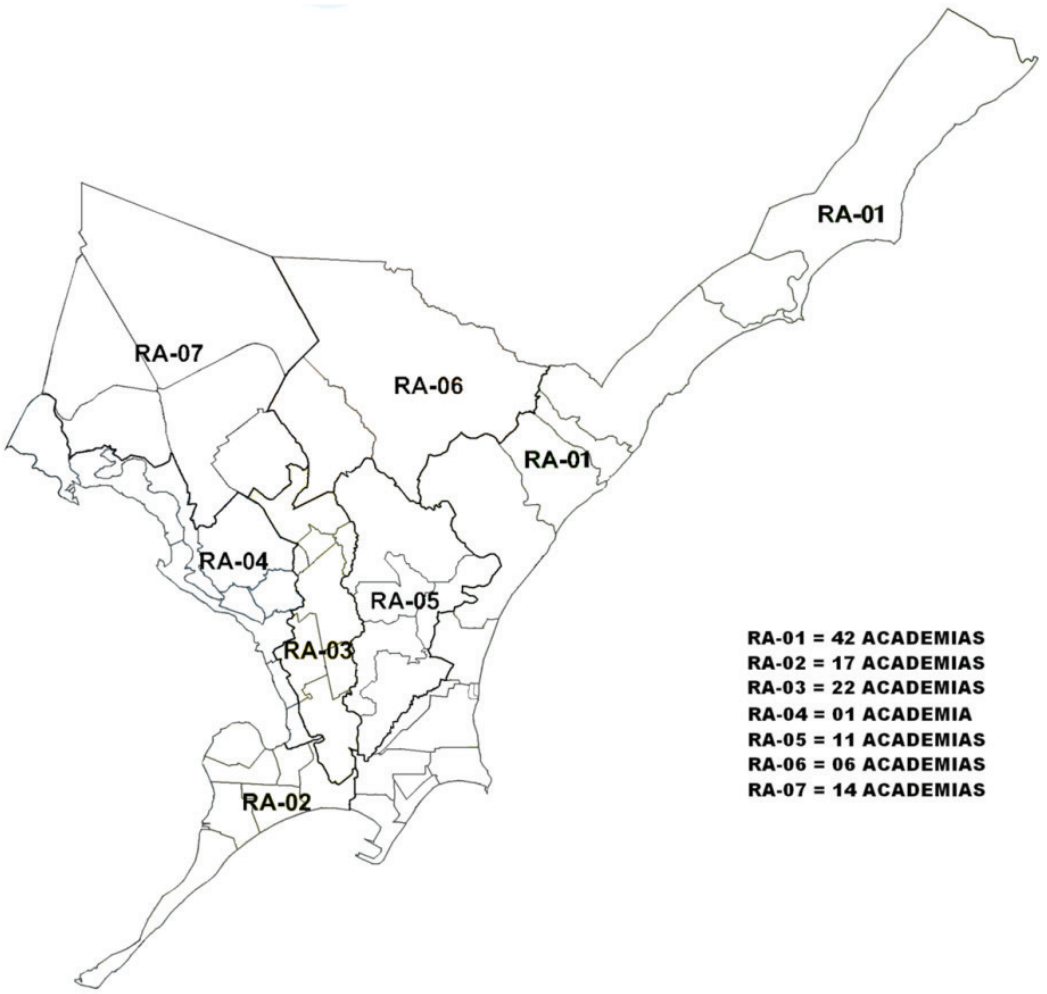

FIGURA 1 - Mapa de Maceió com a distribuição das academias de ginástica de acordo com a região administrativa.

Foi estabelecido que $50 \%$ das academias de cada região administrativa seriam visitadas. A seleção das mesmas em cada região foi feita através de sorteio aleatório. Ao todo participaram do estudo 58 academias. O critério de inclusão foi possuir um programa de avaliação física nas atividades disponibilizadas. Foram excluídas as academias que realizavam apenas medidas antropométricas.

De acordo com Daher et al. ${ }^{\text {, }}$ as avaliações em academias de ginastica seguem uma determinada rotina onde estão presentes os seguintes componentes: anamnese; medidas fisiológicas e antropométricas; avaliação postural; aptidão física relacionada a saúde; emissão dos laudos e interpretação dos dados 
com o aluno para prescrição do programa de exercícios. Nesse sentido foi elaborado um questionário semi-estruturado contendo questões referentes a presença desses itens no programa de avaliação, bem como o instrumento que utilizavam para tal fim. $\mathrm{O}$ instrumento foi avaliado por dois especialistas da área de Educação Física e Medidas e Avaliação. Ao término do preenchimento foi solicitada cópia do formulário utilizado pelas respectivas academias visitadas para um posterior checklist das respostas.

As academias sorteadas foram visitadas num primeiro momento para identificação da presença de um programa de avaliação na sua rotina. Nas regiões administrativas 1(RA-1) e 3 (RA-3), quatro academias não ofereciam avaliação; na região administrativa 2 (RA-2) uma academia não oferecia avaliação e duas se recusaram a participar. Foi então realizado novo sorteio para substituição das mesmas. Após consentimento foi agendada visita ao profissional responsável pela avaliação.

Todos os profissionais avaliadores assinaram o Termo de Consentimento Livre e Esclarecido - TCLE. Esse trabalho foi submetido e aprovado pelo comitê de ética da Universidade Federal de Alagoas sob o n.025347-210-96.

Os dados foram tabulados na planilha eletrônica SPSS (Statistical Package for the Social Sciences) versão 21 e apresentados sob a forma de estatística descritiva: frequência absoluta e relativa. Para verificar associação as diferenças nos programas de avaliação existentes entre academias que têm à avaliação física como obrigatoriedade e àquelas que não obrigam os clientes a fazerem, utilizou-se o teste de Qui-quadrado de heterogeneidade ou o Teste Exato de Fisher em células com frequência menor que cinco. Foi estabelecido nível de significância de $5 \%$.

\section{RESULTADOS}

A distribuição da amostra na tabela 1 apresenta as variáveis referentes à oferta do serviço nas academias. Apesar de todas as academias visitadas oferecerem o serviço de avaliação, nem todas condicionavam a efetivação da matrícula dos alunos a esse serviço. Observou-se que em 27,6\% das academias, o aluno/cliente poderia iniciar o programa de exercícios sem realizar avaliação $(\mathrm{p}=0,005)$. Foi investigada a sistematização para as próximas avaliações e verificou-se que $36,2 \%$ dos estabelecimentos não exigia reavaliação dos usuários.

De acordo com os profissionais das academias analisadas, a motivação estética foi o objetivo que apresentou prevalência semelhante à motivação saúde. Das academias investigadas, a presença do indicador sobre morbidade auto referida apresentou prevalência significativa $(\mathrm{p}<0,005)$; contudo, na proporção de academias que questionaram sobre o histórico clínico da família, não foi observada diferença significativa. Os profissionais também apontaram que as queixas de saúde mais comuns dos usuários estavam distribuídas da seguinte forma: $34,5 \%$ relatava dores no corpo, 31,0\% obesidade, $20,7 \%$ hipertensão/diabetes e $13,8 \%$ outros problemas de saúde. A maioria das academias se preocupava em questionar sobre a ingestão de algum tipo de medicamento $(\mathrm{p}<0,005)$. Diferença significativa também foi encontrada quanto a solicitação de algum tipo de documento clínico declarando as condições de saúde do usuário (Tabela 1).

Em relação aos comportamentos de saúde, o tabagismo e a atividade física, bem como a medida fisiológica (pressão arterial sistêmica) estavam presentes na maioria das avaliações propostas pelas academias $(\mathrm{p}<0,005)$. $\mathrm{Na}$ avaliação 
postural também foi encontrada diferença significativa. Nas medidas relacionadas com a aptidão física, a medida das dobras cutâneas apresentaram presença significativa na maioria das academias. Os componentes flexibilidade e força/resistência muscular localizada (RML), não apresentaram diferença significativa nas rotinas de avaliação das academias $(p=0,079)$ e $(p=0,600)$ respectivamente. A grande maioria das academias não realizava avaliação da resistência cardiorrespiratória nos usuários ( $\mathrm{p}<0,005)$. Após realização da avaliação, as informações eram repassadas predominantemente de modo verbal. (Tabela 1).

TABELA 1 - Distribuição das academias de acordo com os indicadores analisados. Maceió-AL.

\begin{tabular}{|c|c|c|c|}
\hline \multirow{2}{*}{ Indicadores das academias } & Sim & Não & \multirow{2}{*}{ p-valor } \\
\hline & $\%(n)$ & $\%(n)$ & \\
\hline Obrigatoriedade da avaliação & $72,4(42)$ & $27,6(16)$ & $0,005^{*}$ \\
\hline Reavaliação & 63,8 (37) & $36,2(21)$ & 0,079 \\
\hline Objetivo da clientela - Estética & $55,2(32)$ & $44,8(26)$ & 0,600 \\
\hline Objetivo da clientela - Saúde & $44,8(26)$ & $55,2(32)$ & 0,600 \\
\hline Morbidade referida & $70,7(41)$ & $29,3(17)$ & $0,008^{*}$ \\
\hline Histórico familiar de DCNT & $58,6(34)$ & $41,4(24)$ & 0,305 \\
\hline Problemas de saúde - Dores & $34,5(20)$ & $65,5(38)$ & $0,048^{*}$ \\
\hline Problemas de saúde - Obesidade & $31,0(18)$ & $69,0(40)$ & $0,015^{*}$ \\
\hline Problemas de saúde -hipertensão/obesidade & $20,7(12)$ & $79,3(46)$ & $<0,001^{*}$ \\
\hline Ingestão de medicamentos & $72,4(42)$ & $27,6(16)$ & $0,005^{*}$ \\
\hline Tabagismo & $82,8(48)$ & $17,2(10)$ & $<0,001^{*}$ \\
\hline Atividade física & $81,0(47)$ & $19,0(11)$ & $<0,001 *$ \\
\hline Pressão arterial sistêmica & $74,1(43)$ & $25,9(15)$ & $0,002^{*}$ \\
\hline Exames complementares & $70,7(41)$ & $29,3(17)$ & $0,008^{*}$ \\
\hline Avaliação postural & $81,0(47)$ & $19,0(11)$ & $<0,001^{*}$ \\
\hline Dobras cutâneas & $89,7(52)$ & $10,3(06)$ & $<0,001^{*}$ \\
\hline Flexibilidade & $36,2(21)$ & $63,8(37)$ & 0,079 \\
\hline Força/RML & $55,2(32)$ & $44,8(26)$ & 0,600 \\
\hline Resistência cardiorrespiratória & $19,0(11)$ & $81,0(47)$ & $<0,001^{*}$ \\
\hline Disseminação verbal dos resultados & $58,6(34)$ & $41,4(24)$ & 0,305 \\
\hline Disseminação documental dos resultados & $10,3(06)$ & $89,7(52)$ & $<0,001^{*}$ \\
\hline Disseminação verbal e documental & $31,1(18)$ & $68,2(40)$ & 0,018 \\
\hline
\end{tabular}

DCNT: doenças crônicas não transmissíveis; RML: resistência muscular localizada; ${ }^{*} p \leqslant 0,05$ (Teste de comparação entre proporções).

$\mathrm{Na}$ comparação dos serviços prestados pelas academias, que têm avaliação física como obrigatória com as outras que não exigem (Tabela 2), pode-se verificar que aquelas que exigem realização da avaliação antes do inicio do programa de exercício físico, em sua maioria (97,3\%) também exigiam a reavaliação. Em relação aos objetivos dos clientes, das academias que realizavam a reavaliação física, a maior parte tinha como objetivo a saúde, enquanto nas que não exigiam a reavaliação, a motivação maior relatada era a estética $(\mathrm{p} \leq 0,005)$. A flexibilidade foi avaliada na maior parte das academias que tinham avaliação física como exigência, por outro lado, não foi avaliada naquelas que não obrigavam a realização de avaliações físicas $(\mathrm{p} \leq 0,005)$. A investigação das demais variáveis analisadas não apresentou diferenças significantes entre as academias. 
TABELA 2 - Comparação dos indicadores das avaliações físicas entre as academias que exigiam e que não exigiam a realização de avaliação antes do início do programa de exercício físico. Maceió-AL.

\begin{tabular}{|c|c|c|c|}
\hline \multirow{3}{*}{ Indicadores das academias } & \multicolumn{2}{|c|}{ Obrigatoriedade da avaliação } & \multirow{3}{*}{ p-valor } \\
\hline & $\operatorname{Sim}(n=42)$ & Não $(n=16)$ & \\
\hline & $\%(n)$ & $\%$ (n) & \\
\hline \multicolumn{4}{|c|}{ Obrigatoriedade na Reavaliação } \\
\hline Sim & $97,3(36)$ & $2,7(01)$ & $<0,001^{*}$ \\
\hline Não & $28,6(06)$ & $71,4(15)$ & \\
\hline \multicolumn{4}{|l|}{ Objetivo da clientela } \\
\hline Estética & $59,4(19)$ & 40,6 (13) & $0,018^{*}$ \\
\hline Saúde & $88,5(23)$ & $11,5(03)$ & \\
\hline \multicolumn{4}{|l|}{ Morbidade referida } \\
\hline Sim & $75,6(31)$ & $24,4(10)$ & 0,398 \\
\hline Não & $64,7(11)$ & $35,3(06)$ & \\
\hline \multicolumn{4}{|l|}{ Histórico familiar de DCNT } \\
\hline Sim & $70,6(24)$ & $29,4(10)$ & 0,711 \\
\hline Não & $75,0(18)$ & $25,0(06)$ & \\
\hline \multicolumn{4}{|l|}{ Problemas de saúde } \\
\hline Dores & $80,0(16)$ & $20,0(04)$ & 0,123 \\
\hline Obesidade & $55,6(10)$ & 44,4 (08) & \\
\hline Hipertensão/Diabetes & $91,7(11)$ & $8,3(01)$ & \\
\hline Outros & $62,5(05)$ & $37,5(03)$ & \\
\hline \multicolumn{4}{|l|}{ Ingestão de medicamentos } \\
\hline Sim & $69,0(29)$ & $31,0(13)$ & 0,514 \\
\hline Não & $81,3(13)$ & $18,8(03)$ & \\
\hline \multicolumn{4}{|l|}{ Tabagismo } \\
\hline $\operatorname{Sim}$ & $70,8(34)$ & $29,2(14)$ & 0,555 \\
\hline Não & 80,0 (08) & 20,0 (02) & \\
\hline \multicolumn{4}{|l|}{ Atividade física } \\
\hline Sim & $72,3(34)$ & $27,7(13)$ & 0,979 \\
\hline Não & 72,7 (08) & 27,3 (03) & \\
\hline \multicolumn{4}{|l|}{ Pressão arterial sistêmica } \\
\hline Sim & $76,7(33)$ & $23,3(10)$ & 0,212 \\
\hline Não & $60,0(09)$ & $40,0(06)$ & \\
\hline \multicolumn{4}{|l|}{ Exames complementares } \\
\hline Sim & $78,0(32)$ & 22,0 (09) & 0,136 \\
\hline Não & $58,8(10)$ & 41,2 (07) & \\
\hline \multicolumn{4}{|l|}{ Avaliação postural } \\
\hline Sim & 72,3 (34) & $27,7(13)$ & 0,979 \\
\hline Não & $72,7(08)$ & $27,3(03)$ & \\
\hline \multicolumn{4}{|l|}{ Dobras cutâneas } \\
\hline Sim & 71,2 (37) & $28,8(15)$ & 0,527 \\
\hline Não & $83,3(05)$ & $16,7(01)$ & \\
\hline \multicolumn{4}{|l|}{ Flexibilidade } \\
\hline Sim & $57,1(12)$ & $42,9(09)$ & $0,050^{*}$ \\
\hline Não & $81,1(30)$ & $18,9(07)$ & \\
\hline
\end{tabular}




\begin{tabular}{lllc}
\hline Força/RML & & & \\
\hline Sim & $68,8(22)$ & $31,3(10)$ & 0,489 \\
\hline Não & $76,9(20)$ & $23,1(06)$ & \\
\hline Resistência cardiorrespiratória & & & 0,438 \\
\hline Sim & $81,8(09)$ & $18,2(02)$ & \\
\hline Não & $70,2(33)$ & $29,8(14)$ & 0,150 \\
\hline Disseminação dos resultados & & & \\
\hline Verbal & $64,7(22)$ & $35,3(12)$ & \\
\hline Documental & $66,7(04)$ & $33,3(02)$ & $11,1(02)$ \\
\hline Ambos & $88,9(16)$ & & \\
\hline
\end{tabular}

DCNT: doença crônica não transmissível; RML: resistência muscular localizada; ${ }^{*} p \leqslant 0,05$ (Teste qui-quadrado de heterogeneidade).

\section{DISCUSSÃO}

Avaliar atributos relacionados à prática de atividade física é, ou deveria ser, preocupação natural dos profissionais da área da educação física. Nas academias investigadas observou-se uma preocupação com a exigência da avaliação inicial, contudo não foi verificada uma rotina de avaliações periódicas, com exceção daquelas que apresentaram tal exigência inicialmente. Para atender adequadamente às necessidades e aos interesses dos usuários, além das suas características individuais, é também de fundamental importância estar informado sobre as variações individuais no programa de atividade física proposto. Somente assim é possível verificar se houve uma relação positiva entre os estímulos prescritos e os efeitos obtidos, o que só ocorre mediante avaliações periódicas ${ }^{10}$.

Identificar a motivação da clientela é o ponto de partida para elaborar o delineamento de um programa de avaliação. Nesse estudo, estética e saúde tiveram proporção similar. Em revisão da literatura realizada por Liz et al. ${ }^{8}$, sobre os principais motivos de aderência de brasileiros praticantes de exercícios físicos em academias de ginástica, relatou ser as dimensões "estética" e "saúde" como as mais citadas. Apesar da aparência física ser a dimensão associada a esses centros, o fator saúde tem sido apontado como o mais importante para a adesão ${ }^{11}$. Podemos destacar que nas academias que exigiam uma rotina de avaliações, a prevalência de indivíduos com motivação saúde foi significativamente maior. A literatura aponta que indivíduos com idealização da aparência física apresentam maior probabilidade de abandono da prática e menor eficiência na realização dos exercícios físicos ${ }^{12}$. Considerando o aumento da concorrência com a expansão desse mercado de prestação de serviço, talvez esse seja um importante ponto a ser considerado pelas academias que não determinam como obrigatória a avaliação, nem tem a preocupação em organizá-la com indicadores de saúde ${ }^{13}$.

No conjunto de informações esquematizadas no início da avaliação em academias /centros de saúde(anamnese), recomenda-se estarem presentes variáveis que possam identificar o histórico de doenças pessoal e familiar; comportamentos/fatores de risco que fazem parte do cotidiano do indivíduo; uso de medicamentos; queixas e sintomas sugestivos de problemas orgânicos ${ }^{9,14}$. Essas variáveis devem estar organizadas de tal modo que possibilite o profissional estratificar o real perfil das condições de saúde desse indivíduo, inclusive para ver as necessidades de uma avaliação clínica especializada bem como 
exames complementares ${ }^{3}$. Ao observar as variáveis que estão na anamnese do programa de avaliação das academias, identificamos que a grande maioria seguia a recomendação da literatura supracitada. No entanto, através dos formulários de avaliação, não foi possível verificar a comunicação entre essas variáveis nem no sentido de buscar uma estratificação de risco, nem a possibilidade das mesmas serem empregadas para avaliar a evolução da prescrição do programa de exercícios. O cerne de um programa de avaliação é que através da análise dos seus indicadores seja possível tomar decisões quanto a personalização das ações a serem tomadas baseadas em evidências e verificar se essas ações estão tendo impacto positivo.

$\mathrm{Na}$ variável que se refere ao uso de medicamentos, se faz necessário que além ao identificar o uso de algum medicamento, questione-se sobre o tipo e a dosagem, considerando que programas de exercícios físicos podem reduzir sua utilização ${ }^{15}$. Na verificação da pressão arterial, tanto em repouso quanto em movimento, foi verificada prevalência significativa na medida em repouso, contudo é imprescindível que seja estabelecido e cumprido procedimentos básicos para evitar erros de medida nessa importante medida fisiológica ${ }^{16}$. Os comportamentos relacionados à saúde, principalmente tabagismo e atividade física são indicadores que merecem destaque em avaliações das condições de saúde de indivíduos. No âmbito do estilo de vida ativo, atualmente tem se recomendado distinguir os indicadores que se direcionam à promoção da atividade física em si, daqueles que visam reduzir a exposição a comportamentos sedentários ${ }^{17}$. A inatividade física deve ser questionada, principalmente quanto as suas dimensões de lazer e locomoção, e o comportamento sedentário seria o tempo de exposição à: televisão, computador, leitura, videogame, entre outras ${ }^{18}$.

As variáveis que podem ser acrescidas na anamnese de uma academia de ginástica deveriam ser bastante simples e já validadas para determinar as condições de saúde dos indivíduos. Por exemplo, ao serem questionados sobre as principais queixas de saúde dos usuários das academias, os avaliadores apontaram as dores como uma das mais prevalentes. Nesse sentido seria interessante acrescentar ao programa de avaliação instrumentos que possam mensurar o local e a intensidade da dor, considerando ser a prescrição de exercícios físicos defendida há mais de 20 anos pela literatura científica como estratégia para controle da dor ${ }^{19}$. A auto percepção de saúde, indicador representado por uma única pergunta com cinco opções de resposta, pode ser outro importante recurso a ser empregado na anamnese por estar fortemente associado aos aspectos físicos e mentais do individuo, além de ser validado em diferentes culturas $^{20}$. Outro indicador importante citado por alguns avaliadores, mas ausente nos formulários, foi a qualidade de vida, onde as dimensões físicas, psíquicas e sociais podem ser mensuradas sob forma genérica ${ }^{21,22}$.

Nos exames complementares, que inclui a consulta a um médico e os testes por ele solicitados, um correto ordenamento dos indicadores em uma anamnese é que irá determinar a necessidade de um exame clinico e laboratorial complementar ${ }^{3}$. Apesar da maioria das academias atestar esse procedimento, é necessário ficar claro que é o profissional de Educação Física que esta conduzindo a avaliação que identificara essa necessidade, a partir da estratificação das condições de saúde do avaliado.

A correta execução dos movimentos do corpo humano depende de uma boa postura, fato confirmado pela correlação existente entre alinhamento pos- 
tural e desempenho em testes musculares ${ }^{23}$. A grande maioria das academias investigadas realizava algum tipo de avaliação postural no seu programa. Em estudo recente, os autores alertaram para o considerável índice de desvios posturais em praticantes de musculação ${ }^{24}$. Destaca-se que a avaliação postural é um procedimento relativamente simples, contudo, sabendo-se que uma série de adequações e até correções posturais podem ser realizados no próprio ambiente da academia, se faz necessário um rigoroso controle na prescrição e na alteração dos desvios encontrados.

Os componentes da aptidão física relacionados à saúde à saúde estão intimamente associados com a prevenção de doenças e a promoção da saúde ${ }^{25}$. Por ser um conteúdo bastante explorado na formação do profissional de Educação Física, estranhamos os resultados encontrados quanto a mensuração desses componentes, onde apenas a composição corporal, através da medida das dobras cutâneas, apontou presença significativa no programa de avaliação das academias investigadas.

Em relação aos outros componentes da aptidão física relacionada à saúde, podemos afirmar que, debilidade nos componentes força/resistência muscular podem desencadear distúrbios musculoesqueléticos que resultam em dor e desconforto considerável. Por outro lado, indivíduos com musculatura fortalecida são menos expostos a fadigas localizadas e a menor aumento da pressão arterial quando submetidos a esforços físicos mais intensos ${ }^{26}$, além de desempenhar importante papel na regulação hormonal e no metabolismo de alguns substratos, particularmente na sensibilidade insulínica dos tecidos muscula$\mathrm{res}^{27}$. Na flexibilidade, os exercícios de alongamento permitem maior elasticidade dos componentes do aparelho locomotor, propicia maior resistência às lesões, além de reduzir a ocorrência de alterações posturais e menor incidência de dores musculares ${ }^{28} . \mathrm{Na}$ aptidão cardiorrespiratória, onde parece haver uma correlação inversa entre esse componente e a ocorrência de manifestações da doença arterial coronária (DAC) ${ }^{29}$, sua avaliação pode ser importante por identificar o principal componente fisiológico associado à essa aptidão que é o $\mathrm{VO}_{2}$, que além de representar um prognóstico de DAC se mostra importante na elaboração da prescrição para atingir os efeitos benéficos do exercício sobre o $\mathrm{VO}_{2}$ de pico.

Apesar de um estudo realizado em academias de Curitiba-PR apontar que a motivação maior de ingresso dos alunos está no componente da aptidão física composição $\operatorname{corporal}^{30}$, é preciso estar vigilante quanto à adesão desses indivíduos. Nesse sentido é necessário conscientizá-los acerca dos outros componentes e sua relação com os padrões relacionados à saúde, inclusive estabelecendo as normas a serem alcançadas. Acreditamos que o custo de instrumentos para avaliação dos componentes da aptidão física não se constitui numa barreira para sua aquisição e consequentemente implementação.

$\mathrm{Na}$ última etapa do programa de avaliação nas academias, observamos que o rol de informações coletadas são explicadas prioritariamente de modo verbal. Considerando a grande quantidade de indicadores presentes numa avaliação em academias de ginastica, e que manter o estilo de vida ativo requer a compreensão do processo, criação de incentivos e ampliação do conhecimento sobre o serviço, se faz necessário que o aluno tenha informações suficientes para que possa compreender de fato a inter-relação do impacto da prescrição dos exercícios nos diversos indicadores avaliados. Nesse sentido, seria inte- 
ressante, todos os alunos avaliados receberem uma pasta personalizada (como aquela entregue nos laboratórios clínicos) contendo informações do seu estado atual e dos padrões de referência a serem alcançados.

A principal limitação do presente estudo foi seu desenho, o delineamento transversal não permite estabelecer relação de causa e efeito entre a variável obrigatoriedade da avaliação com os outros componentes investigados. Também consideramos como limitação, a não caracterização da formação dos profissionais investigados, bem como, sugerimos que numa futura investigação seja incluída as academias que não tem programas de avaliação e aquelas que a fazem parcialmente. De qualquer forma, esta pesquisa adiciona informações a respeito da rotina das avaliações físicas na literatura nacional que ainda não foram reportadas.

Com os resultados encontrados nesse estudo, considerando as especificidades no âmbito das academias de ginástica, podemos concluir que o que se faz em termos de processo não está sendo ofertado adequadamente, pois apesar da presença dos indicadores recomendados pela literatura, sua qualidade fica prejudicada por não ser encontrada diferença significativa na grande maioria deles, bem como a falta de padronização, o que dificulta obter um referencial de análise para esses indicadores. Recomendamos que o que deve ser feito é reestruturar o programa de avaliação tendo como referência as diretrizes baseadas em evidências na área da saúde, tendo em vista atender a demanda do público alvo que busca o serviço em academias de ginástica, atingindo mesmo aqueles com objetivo estético.

\section{Contribuição dos autores:}

NMO participou na coleta dos dados e elaboração do texto. DASS participou da análise dos dados e contribuiu com a escrita. JJOT idealizou o estudo e participou de todas as etapas desse trabalho.

\section{REFERÊNCIAS}

1. Haskell WL, Lee IM, Pate RR, Powell KE, Blair SN, Franklin BA, et al. Physical activity and public health: updated recommendation for adults from the American College of Sports Medicine and the American Heart Association. Circulation. 2007;116:1081-93.

2. American College of Sports Medicine and American Heart Association. Recommendations for cardiovascular screening, staffing, and emergency policies at health/fitness facilities. Med. Sci Sports Exerc. 1998;30:1009-18.

3. ACSM. Diretrizes do ACSM para os testes de esforço e sua prescrição. 8a ed. Rio de Janeiro: Guanabara Koogan, 2010.

4. Victora CG. Avaliando o impacto de intervenções em saúde. Rev. Bras. Epidemiol. 2002;5:2-4.

5. Hoehner CM, Soares J, Perez DP, Ribeiro IC, Joshu CE, Pratt M, et al. Physical activity interventions in Latin America: a systematic review.Am J Prev Med 2008; 34(3):224-233.

6. IHRSA Global Report 2012. The state of the health club industry.. Disponível em: http://www.ihrsa.org/2012-ihrsa-global-report.Acessado em 02/05/2013

7. Silva MC, Rombaldi AJ, Azevedo MR, Hallal PC. Participação atual e passada em academias de ginástica entre adultos: prevalência e fatores associados. Rev. Bras. Ativ. Fis. Saúde. 2008; 13:28-36.

8. Liz CM, Crocetta TB, Viana MS, Brandt R, Andrade A. Aderência a prática de exercícios físicos em academias de ginástica. Motriz. 2010;16:181-88.

9. Daher DJ, Guiselini M, Ghorayeb N, Dioguardi G. Avaliação cardiovascular préparticipação na academia: aspectos médicos e fisiológicos. Rev. Soc. Cardiol. Estado de São Paulo. 2005;15:105-13. 
10. U.S. Department of Health and Human Services. Physical activity Evaluation Handbook.. Atltanta, GA, EUA, 2002.

11. Tahara AK, Schwartz GM, Silva KA. Aderência e manutenção da prática de exercícios em academias. Rev. Bras. Ciênc. Mov.. 2003; 11:7-12.

12. Luz LS, Silva VAP, Pitanga FJG. Motivos que levam as pessoas a frequentarem um programa de condicionamento físico. Coleção Pesquisa em Educação Física. 2007; 5:377-382.

13. Toscano JJO. Academia de ginástica: um serviço de saúde latente. Rev. Bras. Ciênc. Mov.. 2001; 9:40-42.

14. Balady GJ, Chaitman B, Driscoll D, Foster C, Froelicher E, Gordon N,et al. Recommendations for Cardiovascular Screening, Staffing, and Emergency Policies at Health/Fitness Facilities. Circulation. 1998; 97: 2283-2293.

15. Bertoldi AD, Hallal PC, Barros AJ. Physical activity and medicine use: evidence from a population-based study. BMC public health. 2006; 6:1-6.

16. Pickering TG, Hall JE, Appel LJ, Falkner BE, Graves J, Hill MN, et al. Recommendation for blood presure measurement in humans and experimental animals: Part 1: Blood pressure measurement in humans. Hypertension 2005; 45:142-61.

17. Dietz W. The role of lifestyle in health: the epidemiology and consequences of inactivity. The proceedings of the Nutrition Society. 1996; 55:829-40.

18. Healy GN, Clark BK., Winkle EA. Measurement of Adults' Sedentary Time in Population-Based Studies. Am J Prev Med 2011;41:216-227.

19. Souza JB. Poderia a atividade física induzir analgesia em pacientes com dor crônica? Rev Bras Med Esporte. 2009; 15(2):145-150.

20. Kaleta D, Polanska K, Dziankowska-Zaborszczyk E, Hanke W, Drygas W. Factors influencing self-perception of health status. Cent Eur J Public Health, v.17, n.3, p.122$27,2009$.

21. The Whoqol Group. The World Health Organization Quality of Life Assessment (WHOQOL): position paper from the World Health Organization. Social Science and Medicine. 1995; 41:1403-1409.

22. Toscano, JJO. Qualidade de vida também é atividade física. Maceió: EDUFAL, 2013.

23. Braccialli LMP, Vilarta R. Aspectos a serem considerados na elaboração de programas de prevenção e orientação de problemas posturais. Rev. paul. educ. fís. 2000;14(1):159-71.

24. Baroni BM, Bruscatto CA, Rech RR, Trentin L, Brum LR. Prevalência de alterações posturais em praticantes de musculação. Fisioter. Mov. 2010; 23(1):129-139.

25. Caspersen CJ, Powell KE, Christenson GM. Physical activity, exercise and physical fitness: definitions and distinctions for health-related research. Public Health Rep. $1985 ; 100: 126-3$

26. Cornelissen VA, Fagard RH. Effect of resistance training on resting blood pressure: a meta-analysis of randomized controlled trials. J Hyperten. 2005; 23:251-59.

27. Gippini, A, Mato A, Pazos R, Suarez B, Vila B, Gayoso P, et al. Effect of long term strenght training on glucose metabolism. Implications for individual impact of high lean mass and high fat mass on relationship between BMI and insulin sensitivity. J Endocrinol Investig. 2002;25:520-25.

28. American College of Sports Medicine. Position Stand. The recomended quantity and quality of exercise for developing and maintaining cardiorespiratory and muscular fitness, and flexibility in healthy adults. Med Sci Sports Exerc. 1998;30:975-91.

29. Sui X, Lamonte MJ, Blair SN. Cardiorespiratory fitness and risk of nonfatal cardiovascular disease in women and men with hypertension. Am J Hypertens. 2007; 20:608-615.

30. Filardo RD, Leite N. Perfil dos indivíduos que iniciam programa de exercícios em academias, quanto a composição corporal e aos objetivos em relação a faixa etária e sexo. Rev Bras Med Esporte. 2001;7:57-62.

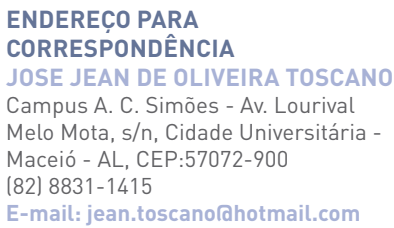

